\title{
Energetics of pupal-adult metamorphosis in the silkworm, Bombyx mori : An analysis of transdeamination parameters in the fat body and haemolymph
}

\section{S. Sivaprasad*}

Department of Zoology, Smt. N.P.S. Government College for Women, Chittoor- 517001 (A.P.), India

E. Bhuvaneswari

Department of Zoology, Smt. N.P.S. Government College for Women, Chittoor- 517001 (A.P.), India

*Corresponding author. E-mail: sivaprasadzoology@gmail.com

\begin{abstract}
Transdeamination seems to be an important alternative energy-intensive gluconeogenesis mechanism that generates glucose from non-carbohydrate sources during pupaladult metamorphosis in Bombyx mori. Studies on four transdeamination parameters, viz., free amino acids (FAA), aspartate aminotransferase (AAT), alanine aminotransferase (AIAT) and glutamate dehydrogenase (GDH) in the fat body and haemolymph of silkworm have indicated that transdeamination shows stage-specific, region-specific and sex -specific variations during metamorphosis. Region-specific growth trends indicate that the transamination reaction, mediated by AAT and AIAT is faster in the abdominal fat body (AFB) and relatively slower in the thoracic fat body (TFB) with concomitant lower FAA levels in the former and higher levels in the latter. Stage-specific growth trends reflect that the energy mobilization through transdeamination assumes greater significance in the early pupal, late pupal and adult stages rather than the mid-pupal stage. Sex-wise trends in FAA levels indicate that the rate of amino acid utilization is significantly faster in females compared to that in males. Further, the growth trends in the levels of GDH vis-àvis aminotransferases signify that the energy demands of male sex expressions are met largely through enhanced levels of $\mathrm{GDH}$ and that the alpha ketoglutarate generated in transamination reaction is used as a substrate for sperm production, sperm motility and successful mating that stimulates fecundity and productivity of in the mulberry silkworm. The study clearly demonstrates that gluconeogenesis through transdeamination supplements the energy requirements of silkworm metamorphosis and that it is facilitated by disintegrating tissues predominantly from the pupal abdominal segments.
\end{abstract}

Keywords: Aminotransferases, Bombyx mori, Free amino acids, Glutamate dehydrogenase, Transdeamination.

\section{INTRODUCTION}

During insect metamorphosis, the larval and pupal tissues are reorganized and restructured into adult organs through a variety of energy intensive processes such as histolysis, histogenesis, differentiation and morphogenesis (Merkey et al., 2011). The fat body, which is actually immersed in the circulating medium of haemolymph, acts as a physiological energy bank and meets the energy demands of dynamic metamorphic events in all insects (Dean et al., 1985). The levels of energy reserves such as glycogen, trehalose and amino acids, accumulated in the fat body during larval stages modulate growth, development and metamorphosis in pupae and sexual maturity in adults (Boggs and Freeman, 2005; Mirth and Riddiford, 2007; Boggs, 2009). Further, it synthesizes a vari-

\section{Article Info}

DOI:10.31018/jans.v10i2.1681

Received: February 28, 2018

Revised: April 21, 2018

Accepted: May 11, 2018

\section{How to Cite}

Sivaprasad, S. and Bhuvaneswari, E. (2018). Energetics of pupal-adult metamorphosis in the silkworm, Bombyx mori : An analysis of transdeamination parameters in the fat body and haemolymph. Journal of Applied and Natural Science, 10(2): 746 - 752 ety of haemolymph proteins, metabolites and antimicrobial peptides apart from detoxifying harmful products of nitrogen metabolism (Keeley, 1985).Biochemical studies on fruit fly and mosquitoes have shown that the fat body specifically expresses amino acid transporters that function as nutrient sensors (Attardo et al., 2005).

The silkworm fat body displays morphological distinctions and works as a region-specific multifunctional organ and mobilizes cellular proteins, amino acids, carbohydrates and lipids as energy reservoirs by integrating signals from neighbouring tissues (Haunerl and Shirk, 1995;Gade, 2004; Seong et.al, 2005; Yaginuma and Ushizima, 2005; Cheng et al., 2006). In this energy-intensive process, some reserve proteins are degraded to individual amino acids, each one of which has its own metabolic destination that generates energy 
(Trivedy et al., 2008; Hemavathi, 2001). Transdeamination, triggered by aminotransferases and glutamate dehydrogenase appears to be one such dynamic metabolic process that drives the proteolytically-derived amino acid pool towards energy production during silkworm metamorphosis (Hemalatha et al., 2014; Sivaprasad, 2015). The present study intends to examine the trends in this energy-intensive process by analyzing metamorphic changes in free amino acids, aminotransferases and glutamate dehydrogenase in the thoracic and abdominal fat bodies and in the circulating medium of haemolymph in Bombyx mori.

\section{MATERIALS AND METHODS}

The Pure Mysore $\mathrm{x} \mathrm{CSR}_{2}$ hybrid strain of $B$. mori was selected as the test species. Biochemical assays were carried out on tissue samples obtained from the thoracic (TFB) and abdominal (AFB) fat bodies and haemolymph of its pupal and adult stages. Earlier, the silkworm larvae were reared under standard environmental conditions of $28^{\circ} \mathrm{C}, 85 \%$ relative humidity $(\mathrm{RH})$ and fed with $M_{5}$ variety of mulberry leaves, 5 times a day at 6 A.M, 10 A.M, 2 P.M, 6 P.M and 10 P.M throughout the larval period (Krishnaswami, 1986). The pupal and adult bodies were dissected mid-dorsally and the fat body samples were collected from the abdominal and thoracic segments separately in Bombyx Ringer (Yamaoka et al., 1971). The haemolymph samples were collected into a prechilled test tube by puncturing the inter-tergal plates of posterior segments. Free amino acid levels were estimated by the method of Moore and Stein (1954) in 5\% homogenates of TFB and AFB samples and in 1:20 diluted haemolymph samples in $10 \%$ trichloroacetic acid (TCA). Both the tissue samples were centrifuged at $2000 \mathrm{rpm}$ for 15 minutes and the supernatant was chosen for the assay. To $0.2 \mathrm{ml}$ of supernatant, $2 \mathrm{ml}$ of ninhydrin reagent was added and heated in a water bath for about 6 minutes, cooled and diluted with $7.8 \mathrm{ml}$ of water. The colour developed was read at $570 \mathrm{~nm}$ in a spectrophotometer against a reagent blank, and the values were expressed in $\mathrm{mg} / \mathrm{gm}$ wet weight of tissue or $1 \mathrm{ml}$ of haemolymph. AAT and AIAT activities were estimated by the method of Reitman and Frankel (1957) in $5 \%$ homogenates of TFB and AFB samples and in $1: 19$ diluted haemolymph samples in distilled water. The homogenates were centrifuged at 600 rpm for 15 minutes and the supernatant was used for the enzyme assay. A reaction mixture of $2.0 \mathrm{ml}$ comprising $100 \mu$ moles of L-aspartic acid or Dalanine, $2 \mu$ moles of ketoglutarate and $0.3 \mathrm{ml}$ of supernatant was prepared and incubated at $37^{\circ} \mathrm{C}$ for 30 minutes and the reaction was stopped by adding $1 \mathrm{ml}$ of 2, 4-dinitrophenyl hydrozine solution $(0.001 \mathrm{M})$ in $0.1 \mathrm{~N}$ hydrochloric acid. After 20 minutes, $10 \mathrm{ml}$ of $0.4 \mathrm{~N}$ sodium hydroxide was add- ed and colour developed was read at $545 \mathrm{~nm}$ in spectrophotometer against a reagent blank. The enzyme activities were expressed as $\mu$ moles of pyruvate formed/ mg protein $/ \mathrm{h}$.

The GDH activity was estimated by the method of Lee and Lardy (1965) in the 5\% homogenates of TFB and AFB samples and in 1: 19 diluted haemolymph samples in ice-cold $0.25 \mathrm{M}$ sucrose solution. The homogenate were centrifuged at 1000 rpm for 15 minutes and the supernatant was used for the enzyme assay. The reaction mixture in a volume of $2 \mathrm{ml}$ contained $100 \mu$ moles of phosphate buffer $\left(p^{H} 7.2\right), 4.0 \mu$ moles of sodium glutamate, $0.1 \mu$ moles of nicotinamide adenine dinucleotide , 4 umoles of 2-4 iodophenyl -3, (4 nitrophenyl)-5 phenyl tetrazolium chloride (INT) and $0.2 \mathrm{ml}$ of supernatant was prepared and incubated at $37^{\circ} \mathrm{C}$ for 30 minutes. The reaction was stopped by adding $5 \mathrm{ml}$ glacial acetic acid and the formazone formed was extracted overnight in $5 \mathrm{ml}$ of cold toluene. The intensity of colour developed was read against a toluene blank at $495 \mathrm{~nm}$ in a spectrophotometer and the enzyme activity was expressed as $\mu$ moles of formazone formed $/ \mathrm{mg}$ protein/h. The experimental data were statistically analyzed using online software (http:// www.graphpad.com/ quick calcs/ index cfm http:// www.percent-change.com/index.php?

y1=101andy2=7800) and MS Excel platforms and the growth trends in all parameters were analyzed in terms of compound periodical growth rates (CPGR's) as given by Sivaprasad (2012).

\section{RESULTS}

Transitional changes in the levels of transdeamination parameters during pupal-adult metamorphosis in $B$. mori are presented in Table 1 and Figs. 1 to 3 .

Free amino acids: The levels of free amino acids in the thoracic (TFB) and abdominal (AFB) fat bodies have shown increasing growth trends in early phases and declining growth trends in later phases of pupal development (Table 1; Cols.3,4). The analysis of free amino acid data in terms of compound periodical growth rates (CPGRs) has shown that their levels grew @6.49\% per day in the TFB and @18.97\% in the AFB, indicating their availability for transdeamination from early to mid pupal stage. However, their levels declined @ $28.37 \%$ per day in TFB and @ $40.31 \%$ per day in AFB during the second half of the pupal life (i.e., from day 5 to day 9). Further, the declining trend in FAA levels continued through pupal-adult transition in a sex specific fashion. In TFB, their levels declined by $80.11 \%$ in males and $90.77 \%$ in females and in AFB it did so by $35.43 \%$ in males and $85.75 \%$ in females, reflecting the corresponding proportions of their utilization in both the sexes (Table 1,Cols.3-4).By and large, similar upward and down-ward trends in FAA levels were ob- 


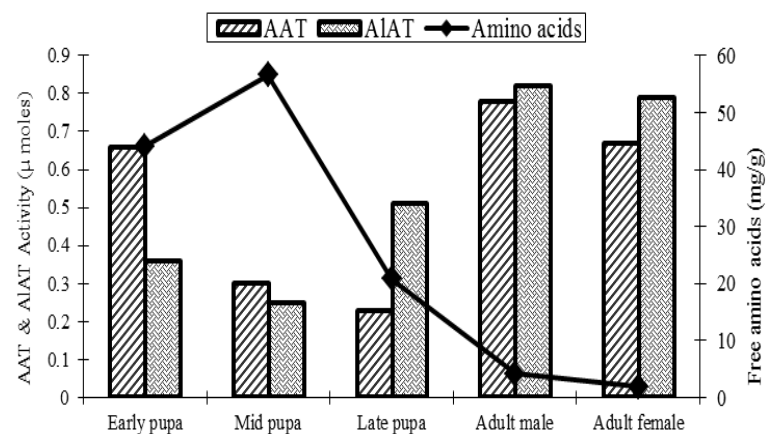

A. Thoracic fat body (TFB)

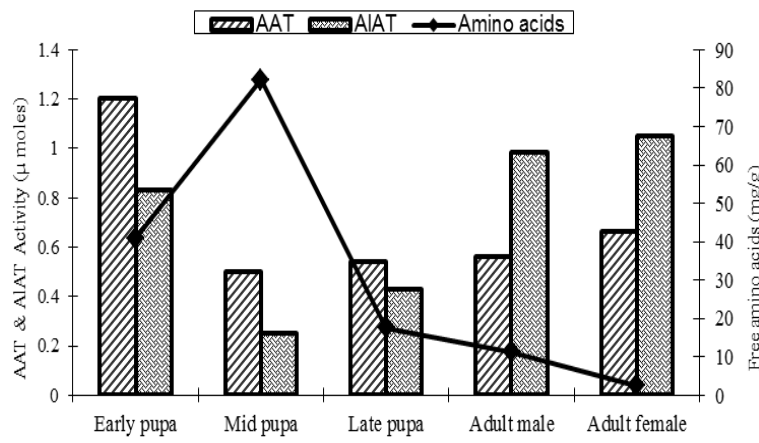

B. Abdominal fat body $(A F B)$

Fig. 1. Free amino acids (FAA) versus aminotransferases (AAT and AIAT)) in the fat body of $B$. mori during pupal-adult metamorphosis.

served in the circulating medium of haemolymph during pupal-adult transition, wherein their levels were elevated @8.79\% per day during the first half of pupal life (from day 1 to day 5 ), but, declined thereafter insignificantly $(<1 \%)$ during the second half of pupal life (day 5 to day 9). Nevertheless, amino acid levels were elevated by $64.5 \%$ in males and $73.98 \%$ in females during pupal transformation (Table 1; Col. 5).

AAT activity: In general, the activity levels of aspartate amino transferase (AAT) in the fat body projected declining trends during pupal life and elevatory trends during pupal-adult transition (Table 1; Cols. 6, 7). From early to mid pupal life, the enzyme activity recorded negative CPGRs both in the TFB $(-17.89 \%)$ and AFB $(-19.66 \%)$ and the declining trend was extended tolate pupal stage (@-8.48\% per day) in the former, notwithstanding its slight elevation (@ 2.6\% per day) in the latter. Surprisingly the downward trend in AAT activity got reversed suddenly and peaked to new heights during pupal-adult transition. A dramatic increase in the activity levels of this transferase was observed in TFB compared to that in AFB. While in TFB, its activity grew maximally by $239.13 \%$ in males and by $191.3 \%$ in females, in AFB it recorded a moderate growth rates of $3.7 \%$ and $22.22 \%$ in both the sexes respectively (Table 1 , Cols 6,7$)$. In the haemolymph, its activity maintained a static level during the first half of pupal life (i.e. from day 1 to day 5), but slumped @9.14\% 


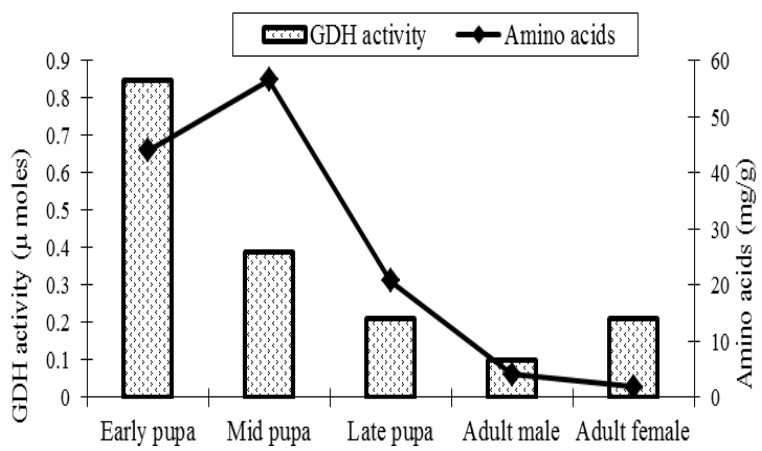

A. Thoracic fat body (TFB)

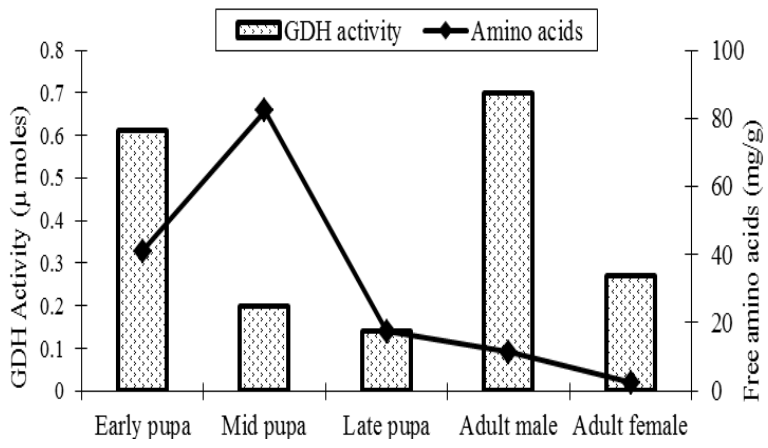

B. Abdominal fat body (AFB)

Fig. 2. Free amino acids (FAA) versus glutamate dehydrogenase $(G D H)$ in the fat body of $B$. mori during pupal-adult metamorphosis.

per day during the latter half of the pupal metamorphosis (i.e. from day 5 to day 9). Nevertheless, its activity peaked to new heights both in males $(33.33 \%)$ and females $(66.67 \%)$ during the pupal-adult transition (Table 1; Col. 8).

AIAT activity: By and large, the metamorphic changes in the activity levels of alanine amino transferase (AIAT) were similar to those of AAT. Its activity declined @ 8.71\% per day in the TFB and @25.92\% per day in the AFB, indicating slower transamination during the first half of the pupal life. But, its activity levels showed impressive upward trends thereafter, recording an elevation @26.83\% per day in TFB and @19.81\% per day in AFB, reflecting faster transamination in the later stages of pupal life. The growth trends in enzyme activity continued although during pupaladult transition with a male biased approach. It grew by $60.78 \%$ in TFB and $127.91 \%$ in AFB in males and by $54.94 \%$ in TFB and 83.72 in AFB in females (Table 1; Cols. 9, 10). However, in the circulating medium of haemolymph, this transferase activity recorded contradictory growth trends during the previous half and complementary trends in the latter half of pupal life. It recorded positive growth rates (CPGR: $+91.09 \%$ ) during the first half and negative growth rates (CPGR: $57.83 \%$ ) during the second half of pupal life. But, in consonance with raising trends in TFB and AFB, the haemolymph transferase recorded positive CPGRs in both males (33.33\%)and females

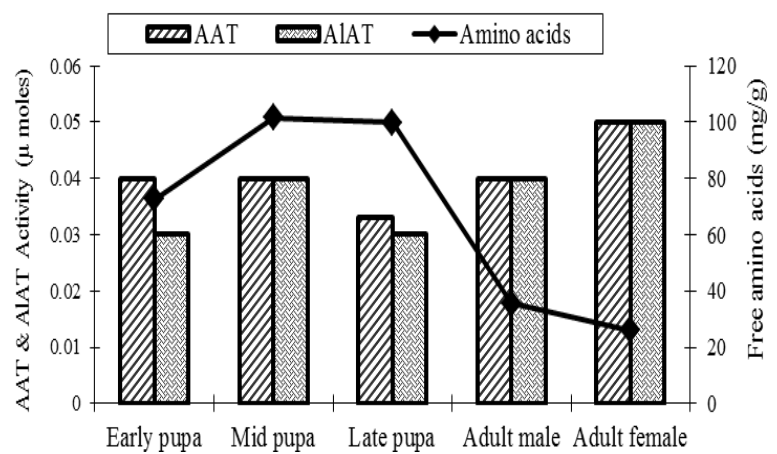

A. FAA and aminotransferases.



\section{B. FAA and $G D H$.}

Fig. 3. Free amino acids (FAA) versus aminotransferases (AAT, AIAT) and GDH in the haemolymph of $B$. mori during pupal-adult metamorphosis.

$(66.67 \%)$ during pupal-adult transition (Table 1; Col. 11).

GDH activity: The fat body GDH recorded negative CPGRs throughout the pupal life in both thoracic and abdominal segments. Its activity declined @17.70\% per day in TFB and @24.33 per day in AFB from early to mid pupal stage (day 1 to day 5 ), but slumped @18.64\% per day in TFB and @11.21\% in AFB from the mid to late pupal stage (Table 1; Cols.12, 13). Interestingly, during pupaladult transition the GDH activity projected sexual and regional disparities with contradictory growth trends. In males, its activity slumped by $52.38 \%$ in TFB but shot-up phenomenally by $400 \%$ in AFB. However in females, its activity remained unchanged in TFB, but showed normal growth rate (CPGR: $92.86 \%$ ) in AFB. In haemolymph, its activity recorded an upward growth trend (CPGR: $+20.74 \%$ ) during the first half of pupal life and a downward growth trend (CPGR: $-29.33 \%$ ) thereafter during its latter half. However, during pupaladult transition, the down fall in its activity continued in males (CPGR:-50\%), but remained unchanged in females (Table 1; Col.14).

\section{DISCUSSION}

The pupal and adult stages represent two nonfeeding stages in the life cycle of $B$. mori. The energetics of pupal-adult transition is an interesting aspect of entomological research. It has been widely acknowledged that the energy demands of 
this transitory phase in insects are met exclusively from the products of intermediary metabolism involving significant turnover in the profiles of carbohydrates, glycogen reserves, proteins, lipids, amino acid and nitrogen within the fat body (Inagaki and Yamashita, 1986; Arrese and Soulages, 2010). It is likely that the carbohydrate reserves deplete fast as they are actively metabolized for energy production during metamorphosis, leaving the silkworm with limited opportunities. Obviously, $B$. mori is forced to explore alternative noncarbohydrate energy sources through amino acid catabolism during non-feeding metamorphic stages. In this process aminotransferases serve as a strategic link between carbohydrate and protein metabolisms (Saravanan et al.,2011). Thus, transdeamination seems to be an important alternative gluconeogenesis mechanism that drives energydependent metamorphic events in this holometabolous insect. This of course, involves enzymatic conversion of glucogenic amino acids to carbohydrates by the active participation of aspartate amino transferase (AAT), alanine amino transferase (AIAT) and glutamate dehydrogenase (GDH). The modus operandi of transdeamination in silkworm is not clear. Nevertheless, it is presumed to occur in two sequential steps (Scott et al., 2004). The first step involves a transamination reaction mediated by two aminotransferases (AAT and AIAT),resulting in the transfer of amino group of one amino acid to keto acid to generate another amino acid and subsequent collection of such amino groups in the form of L-glutamate. In the second step, L-glutamate is converted to alphaketo glutarate by a mitochondrial matrix enzyme called glutamate dehydrogenase $(\mathrm{GDH})$, which is then channelized through citric acid cycle for the synthesis of glucose and energy release (Voet et al., 1999; Saravanan et al., 2011).

The sources of such non-carbohydrate raw materials for transdeamination have not been traced so far. However, it is presumed that the duel metamorphic events of histolysis and histogenesis are closely synchronized through transdeamination in order to accomplish this energy-rich metabolic feat, during which the disintegrating tissues such as the intersegmental muscle, silk gland, integument and gut contribute free amino acid pool, rich in gluco-neogenic amino acids like aspartate, alanine and glutamate (Trivedy et al., 2010; Paijo, 2010; Sivaprasad and Sailaja, 2010;Sivaprasad, 2015; Aparupa, 2015). The fat body collects such amino acids from the circulating haemolymph and metabolizes them through appropriate energyintensive pathways during metamorphosis, morphogenesis and egg maturation in insects (Keeley, 1985). The current study on the transdeamination parameters in the fat body vis-à-vis the haemolymph amply demonstrates the prevalence of such a mechanism in B. mori. In this feat, the silkworm fat body functions as a distinct structural and functional organ in the thoracic and abdominal segments and these two regions are connected biochemically and physiologically by the circulating medium of haemolymph. As demonstrated in our previous study with reference to intersegmental muscle (Sivaprasad, 2015), the fat body of silkworm, displays region-specific, stagespecific and sex-specific growth trends in all the transdeamination parameters examined.

Region specificity: The region-wise metamorphic trends indicate that the transamination, mediated by AAT and AIAT is faster in AFB buts lower in TFB. This is substantiated by the prevalence of higher FAA levels in TFB and correspondingly lower levels in AFB (Figs. 2A, 2B). Further, as observed in the present investigation, the opposing trends in the activity levels of two aminotransferases (AAT, AIAT) vis-à-vis FAA levels, amply demonstrate that the free amino acid reserves are slowly metabolized (CPGR: $-28.37 \%$ ) in the thoracic segments and actively(CPGR: $-40.31 \%$ ) metabolized in the abdominal segments (Table 1; Cols.3,4). Obviously, in holometabolous insects like silkworm, the AFB plays greater role in meeting the energy requirements of pupal metamorphosis through transdeamination, more rapidly than that that of TFB. Nonetheless, the latter supplements the energy needs of metamorphosis uninterruptedly in a slow and steady state. As reported in our earlier findings, the transdeamination pathway seems to be more active in degenerating tissues compared to those of reforming ones (Sivaprasad, 2014). Evidently, the rapidly degenerating intersegmental muscle tissue and quickly remodeling fat body cells supply the much needed free amino acid reserves for transdeamination in the AFB. Conversely, the slower utilization of FAA reserves in transdeamination in the thorax indicates that they might have greater role in the formation of thoracic capsule and in the synthesis of additional proteins required for the growth and development of musculature associated with wings and walking legs during pupal-adult transformation (Sivaprasad, 2015). In the whole event, the haemolymph shuttles FAA and enzymes between thorax and abdomen as per metamorphic energy demands as reported by Sabhat et al., 2013. The complementary growth trends in all the transamination parameters observed in this fluid medium amply demonstrates this fact (Fig. 3A. 3B).

Stage specificity: The growth trends in the levels of transdeamination parameters denote that energy mobilization through transdeamination process assumes greater significance during the periods of high demand prevalent in the early pupal, late pupal and adult stage of silkworm (Sabhat et al., 2013). Apparently, the activity levels of all the three enzymes (i.e. AAT, AIAT and GDH), which maintained a higher profile during the early pupal 
stages actually declined in the mid and late pupal stages and increased in the adult stages (Figs. $1 \mathrm{~A}, 1 \mathrm{~B}, 2 \mathrm{~A}, 2 \mathrm{~B})$. These fluctuating trends reflect the prevalence of similar ups and downs in the pathway of gluconeogenesis and energy release during insect metamorphosis (Bharathi and Sucharitha, 2006; Burrows, 2007). More particularly, the additional energy requirements of body reorganization and reformation that characterize larvalpupal and pupal-adult transition stages are either partially or predominantly met by boosting gluconeogenesis through transdeamination. More elaborate studies are needed to prove conclusively the existence of such a biochemical transitional mechanism during silkworm metamorphosis.

Sex specificity: The study demonstrates clearcut sexual disparity in transdeamination parameters. The negative CPGRs, caused due to depletion of FAA levels indicate their rapid utilization in females (CPGR: -85.75 to $-90.77 \%$ ) compared to males (CPGR: -35.43 to $-80.11 \%$ ). The sexspecific nature of transdeamination reaction is closely linked to regional specificity; wherein the amino acid turnover is more pronounced in thorax (CPGR: 80.11 to $90.77 \%$ ) than that in abdomen (CPGR: 35.43 to $85.75 \%$ ). Obviously, transamination reaction is preferentially mediated by different transferases in different regions; AAT in thorax and AIAT in abdomen (Fig. 2A). Interestingly, the FAA pool metabolized by GDH is limited to one sex as evidenced by explosion in its activity (CPGR: $400 \%$ ) in males, while it carried through significantly on a low sustained note (CPGR:92.86\%)in females (Table 1 ;Col.13;Fig.2B). Such a sex-biased explosion in GDH activity clearly substantiates that the energy demands of male sex and its expression are met through enhanced levels of GDH and that the alpha ketoglutarate generated in transamination reaction is used as a substrate for sperm production, sperm motility and successful mating that stimulates fecundity and productivity of in B. mori (Hemavathi, 2001; Bharathi and Sucharitha, 2006; Hemalatha et al., 2014). Needless to say most of such energy requirements are met through active transdeamination in the AFB rather than in TFB.

\section{Conclusion}

It has been demonstrated that the transdeamination represents an alternative source of energy for pupal-adult metamorphosis in $B$. mori, during which glucose is synthesized from the glucogenic amino acids emanating from the disintegrating larval tissues such as the fat body and muscle in a stage-specific, region-specific and sex-specific manners (Sivaprasad, 2015). This process takes active part in the abdominal segments compared to that of thorax. The study further substantiates that transdeamination is very active in the abdomen; because its tissues undergo total transfor- mation in form and function during pupal-adult metamorphosis and that the amino acid pool for this alternative energy metabolism is principally derived from the AFB that undergoes remodeling during metamorphosis. Further, the growth trends in the parameters of transdeamination indicate that the mobilization glucogenic amino acids occur through haemolymph in a sex-specific pattern. In females it is mediated by aminotransferases (AAT and AIAT), while it is carried through by $\mathrm{GDH}$ in males. By storing or releasing components central to all events of reproduction and by suitably modulating the transdeamination reaction in the thoracic and abdominal segments, the fat body coordinates silkworm growth and reproduction. In order to perform these multiple metabolic functions and to fulfill the changing physiological needs of metamorphosis, the fat body integrates signals from other organs through the transporting medium of haemolymph (Gade, 2004; Mirth and Riddiford, 2007; Sabhat et al., 2013).

\section{ACKNOWLEDGEMENTS}

The Department of Science and Technology, New Delhi, India is acknowledged for providing the financial support in the form of Research Project (SR/SO/AS-76/2010, Dt.01.02.2012) to Dr. S. Siva Prasad.

\section{REFERENCES}

Aparupa, B. (2015). Silk and its biosynthesis in silkworm Bombyx mori. J. Academia and Ind. Res, 3 (12): 626628.

Arrese,E.L.and Soulages, J.S. (2010). Insect Fat Body: Energy Metabolism and Regulation. Entomology,55: 207-225.

Attardo, G.M., Hansen, I.A. and Raikhel, A.S. (2005). Nutritional regulation of vitellogenesis in mosquitoes: implications for an autogeny. Insect Biochem Mol Biol, 35:661-75.

Bharathi, D. and Sucharitha, K.V. (2006). Impact of prolactin on day-to-day changes in the protease activity in the mid gut of fifth instar silkworm, Bombyx mori $\mathrm{L}$. Ind.J.Com, Anim. Physiol. 24(1):42 -46.

Burrows, M.(2007). Anatomy of the hind legs and action of their muscles during jumping in leafhopper insects. J. Exp. Biol. 210: $3590-3600$.

Boggs, C.L. (2009). Understanding insect life histories and senescence through a resource allocation lens. Functional Ecology. 23: 27-37.

Boggs,C.L. and Freeman, K.D. (2005). Larval food limitation in butterflies: effects on adult resource allocation and fitness. Ecologia. 144: 353-361.

Cheng, D.J., Xia, Q.Y., Zhao, P., Wang, Z.L. and Xu, H.F.(2006). EST-based profiling and comparison of gene expression in the silkworm fat body during metamorphosis. Arch Insect Biochem Physiol. 61:10-23.

Dean, R.L., Collins, J.V. and Locke, M. (1985). Structure of the fat body. Comprehensive Insect Physiology, Biochemistry and Pharmacology (Editors: Kerkut, G.A. and Gilbert, L.I.) Pergamon, New York. pp. 155210.

Gade, G. (2004). Regulation of intermediary metabolism 
and water balance of insects by neuropeptides. Annu Rev Entomol. 49:93-113.

Haunerl and, N.H. and Shirk, P.D. (1995). Regional and functional differentiation in the insect fat body. Annu Rev Entomol. 40:121-45.

Hemalatha, A., Bhuvaneswari, E., Sivaprasad,S. and Yellamma, K. (2014).Metamorphosis-triggered transdeamination of amino acids in the silkworm, Bombyx mori. Ind. J. Appl., Res.4 (11): 475-478.

Hemavathi, B. (2001). Effect of thyroxine on growth and metabolic activities of silkworm, Bombyx mori L. Ph. D. Thesis. Sri Padmavati MahilaVisvavidyalayam, Tirupati, A.P, India.

Inagaki, S. and Yamashita, O. (1986). Metabolic shift from lipogenesis to glycogenesis in the last instar larval fat body of the silkworm, Bombyx mori. Insect Biochem.16:327-31.

Keeley, L.L. (1985). Biochemistry and physiology of the insect fat body. Comprehensive Insect Physiology, Biochemistry and Pharmacology (Editors: Kerkut, G.A.and Gilbert, L.I.), Vol. 3. Pergamon, New York, pp 211-28.

Krishnaswami, S. (1986). New technology of silkworm rearing. Central Sericultural Research and Training Institute, Mysore, India.

Lee,Y.P. and Lardy, H.A.(1965). Influence of thyroid hormones on phosphate dehydrogenase and other dehydrogenases in various organs of the rat. J. Biol. Chem.240: 1427-32.

Mathew, K.E., Van. H. and Ahern, K.G. (2003). Biochemistry, Third Edition, Pearson Education (Singapore) Pvt. Ltd, pp 743.

Merkey, A.B., Wong, C.K., Hoshizaki, D.K. and Gibbs, A.G. (2011). Energetics of metamorphosis in Drosophila melanogaster. J. Insect Physiol., 57 (2011): 1437-1445.

Mirth, C.K. and Riddiford, L.M. (2007). Size assessment and growth control: how adult size is determined in insects? Bio Essays, 29:344-55.

Moore, S. and Stein,W.A. (1954).A modified ninhydrin reagent for the photometric determination of amino acids and related compounds. J. Biol. Chem. 211: 907-913.

Paijo, S. (2010). 'Fat body'. Retrieved December, 20 2010 from www.insectspedia.blogspot.com/2010/10/ fat-body.html.

Reitman, S. and Frankel, S. (1957). A calorimetric method for the determination of serum glutamic-oxalo acetic and glutamic-pyruvic transaminases.Am. J. Clin. Pathol.28:56.

Sabhat, A., Malik, M.A., Kamili, A.S., Malik, G.N., Mir, S.A. and Malik. F.A. (2013). Effect of mulberry nutri- tion on protein and free amino acid levels of haemolymph of selected races of the silkworm, Bombyx mori L.under temperate climates of Kashmir. Asian J. Bio. Sci, 8 (1). 47-51

Saravanan, M., Selvi, S., Veeranarayanan, M., and Saravanan, N. (2011). Modulations in the haemolymph of silkworm (Bombyx mori L (Lepidoptera: Bombycidae)] fed with mulberry leaves augmented with cowpeas (Vigna unguiculata). Int. J. Nutr. Pharmacol. Neurol Dis, 1 (1): 64-68

Scott, R.C., Schuldiner, O. and Neufeld, T.P. (2004). Role and regulation of starvation-induced autophagy in the Drosophila fat body. Dev. Cell. 7: 167-178.

Seong, S.L., Park, K.E., Nagata, M., and Yoshitake, N. (2005). Effect of metamorphosis on the major haemolymph proteins of the silkworm, Bombyx mori. Arch. Insect Biochem. and Physiol.2 (1): 91- 104.

Sivaprasad, S. (2012). Simple method for calculation periodical growth rates in animals and plants. J. Bio. Innov. 5:114-119.

Sivaprasad, S. (2014).Proteolysis-triggered muscular atrophy in the abdominal segments of the silkworm, Bombyx mori during pupal-adult metamorphosis. Ind. J. Appl. Res.,4(3): 504-508.

Sivaprasad, S. (2015). Metamorphic changes in the profiles of transdeamination parameters in the intersegmental muscle of the silkworm, Bombyx mori. Int. J. Adv. in Pharmacy, Biology and Chemistry. 4(4): 1-7

Sivaprasad,S.and Bhuvaneswari, E. (2015). Changes in the levels of proteolytic parameters in the fat body and haemolymph of Bombyx mori during pupal-adult metamorphosis. J.Bio.Innov.4 (5): 180-191.

Sivaprasad, S. and Sailaja, B. (2010). Mobilization and utilization of proteins derived from the disintegrating gut in the silkworm, Bombyx mori during pupal- adult metamorphosis. Int. J. Biol. Sci.1:33-40.

Trivedy, K., Kumar, S.N., Mondal, M. and Bhat, A.K. (2008). Protein banding pattern and major amino acid component in de-oiled pupal powder of silkworm, Bombyx mori. J. Entom. 5:10-16.

Voet, D., Voet, J.G.and Pratt, C.W. (1999). Fundamentals of Biochemistry, John Wiley and Sons, Inc., USA, pp 616-619.

Yaginuma, T. and Ushizima, M. (2005). Proteolytic activity in the fat body during the pupal - adult metamorphosis of the silkworm, Bombyx mori. Exp. Zool. 259 (2): 145-153.

Yamaoka, K., Hoshino, M. and Hiral, T. (1971). Role of Sensory hairs on the anal papillae in position behaviour of Bombyx mori. L Insect Physiol. 47: 2327-2336. 